\title{
Concentrações plasmáticas de paroxetina em pacientes adultos e idosos com depressão
}

\author{
Paroxetine plasma concentrations in adult and elderly depressed patients
}

\author{
Carlos Augusto de Mendonça Lima1, Pierre Baumann², Chin Bin Eap ${ }^{3}$ \\ ${ }^{1}$ Doutor. Professor, Instituto de Psiquiatria, Universidade Federal do Rio de Janeiro (UFRJ), Rio de Janeiro, RJ. ${ }^{2}$ Doutor. Privat docent. Diretor, \\ Unidade de Bioquímica e de Farmacologia Clínica, Departamento Universitário de Psiquiatria, Universidade de Lausanne (UNIL), Lausanne, Suíça. \\ ${ }^{3}$ Doutor. Bioquímico, Unidade de Bioquímica e de Farmacologia Clínica, Departamento Universitário de Psiquiatria, UNIL.
}

\begin{abstract}
Resumo
Introdução: O efeito do envelhecimento nas concentrações plasmáticas em estado de equilíbrio da paroxetina foi investigado em 136 pacientes com depressão, tratados com doses variando entre 10 e $120 \mathrm{mg} /$ dia de paroxetina.

Métodos: Os pacientes foram separados em três grupos: com idade até 64 anos (idade média \pm desvio padrão: 41,7 $\pm 12,6$ anos; $n=44$ ), entre 65 e 79 anos $(72,8 \pm 4,7$ anos; $n=64)$, e com mais de 80 anos $(82,8 \pm 3,3$ anos; $n=28)$. As doses de paroxetina foram normalizadas para 20 $\mathrm{mg} /$ dia. Amostras de sangue foram coletadas em condições de estado de equilíbrio. Os níveis plasmáticos de paroxetina foram medidos por cromatografia gasosa - espectrometria de massa. As funções hepática e renal foram medidas por testes laboratoriais clínicos estandardizados. Resultados: Uma grande variabilidade interindividual dos níveis plasmáticos de paroxetina (37 vezes) foi medida para uma mesma dose. A média dos níveis plasmáticos de paroxetina corrigida para uma dose diária de $20 \mathrm{mg}$ foi $87 \%$ maior no grupo com mais de $80 \operatorname{anos}(56,4 \pm 64,1 \mathrm{ng} / \mathrm{mL} ; \mathrm{p}<0,05)$ e $57 \%$ maior no grupo com idades entre 65 e 79 anos $(46,7 \pm 33,4 \mathrm{ng} / \mathrm{mL} ; \mathrm{p}<0,001)$ quando comparada com a média dos pacientes com menos de 64 anos $(29,9 \pm 11,9 \mathrm{ng} / \mathrm{mL})$. A idade foi correlacionada significativamente com os níveis plasmáticos de paroxetina $(\mathrm{r}=0,21, \mathrm{p}<0,05)$.
\end{abstract}

Conclusão: A redução de doses da paroxetina em pacientes idosos parece justificada.

Descritores: Paroxetina, idosos, depressão, concentração plasmática, antidepressivos.

\begin{abstract}
Introduction: The effect of aging on steady-state plasma concentrations of paroxetine was investigated in 136 depressive patients treated with paroxetine $10-120 \mathrm{mg} /$ day.

Methods: The patients were divided into three groups: aged up to 64 years (mean age \pm standard deviation: $41.7 \pm 12.6$ years; $\mathrm{n}=44$ ), between 65 and 79 years $(72.8 \pm 4.7$ years; $n=64)$, and 80 years or older $(82.8 \pm 3.3$ years; $n=28)$. Paroxetine doses were normalized to $20 \mathrm{mg}$ /day. Blood samples were collected under steady-state conditions. Paroxetine plasma levels were measured by gas chromatography/mass spectrometry. Hepatic and renal functions were measured by standardized clinical laboratory tests.

Results: A large interindividual variability of paroxetine plasma levels (37-fold) was measured for a given dose. The mean plasma levels of paroxetine corrected for a $20 \mathrm{mg}$ daily dose were $87 \%$ higher in the very elderly ( $\geq 80$ years) patients $(56.4 \pm 64.1 \mathrm{ng} / \mathrm{mL} ; \mathrm{p}$ $<0.05)$ and $57 \%$ higher in the elderly $(65-79$ years) patients $(46.7 \pm 33.4 \mathrm{ng} / \mathrm{mL} ; \mathrm{p}<0.001)$ when compared to the adult patients $(<64$ years) $(29.9 \pm 11.9 \mathrm{ng} / \mathrm{mL})$. Age correlated significantly with paroxetine plasma levels $(\mathrm{r}=0.21, \mathrm{p}<0.05)$.
\end{abstract}

Conclusion: Recommended dose reduction of paroxetine in elderly patients seems therefore justified.

Keywords: Paroxetine, elderly, depression, plasma levels, antidepressants.

\section{Introdução}

A paroxetina (PARO), uma fenilpiperidina transisomérica, é um inibidor seletivo da recaptura da serotonina (ISRS), sendo um antidepressivo usado extensivamente $^{1,2}$. Quando comparada com outros antidepressivos ISRS, a PARO é de duas a 23 vezes mais potente ${ }^{3}$. Ela pode ser administrada em dose

\section{Correspondência:}

Instituto de Psiquiatria, CDA Faculdade de Medicina, Universidade Federal do Rio de Janeiro, Rua Aiuru, 63/101, Humaitá, CEP 22261-110, Rio de Janeiro, RJ. E-mail: climasj@yahoo.com 
única, entre 20 e $50 \mathrm{mg}$. Seu metabolismo durante a primeira passagem hepática é de aproximadamente $60 \%$. O pico plasmático ocorre entre 2 e 8 horas após a administração ${ }^{4}$. Ela é biotransformada pelo fígado em metabólitos inativos. Sua alta afinidade com a isoenzima CYP2D6 do citocromo P450 sugere que ela pode interagir com outras substâncias ${ }^{5-8}$.

Seu uso em idosos provou ser tão eficaz quanto outros antidepressivos, com um perfil de efeitos indesejáveis comparável ao seu uso em adultos9. A PARO foi incluida na lista dos antidepressivos recomendáveis para os idosos proposta numa declaração de consenso de peritos ${ }^{1}$. Seu uso por pacientes idosos com depressão reduz significativamente o número de recaídas e de recidivas $^{2}$. Apesar disso, existem poucos estudos farmacocinéticos da PARO nesse grupo de pacientes, em particular aqueles que incluam pessoas com mais de 80 anos.

Observou-se, no estudo de Lundmark et al. ${ }^{10}$, que nos 21 pacientes idosos existe uma considerável variabilidade interindividual dos seus níveis plasmáticos no estado de equilíbrio e da meia-vida de eliminação. Os autores consideraram que esses resultados eram insuficientes para justificar uma redução da dose da PARO em idosos. Esta mesma grande variabilidade interindividual dos níveis plasmáticos foi confirmada por Sindrup et al., que observaram uma variação de 25 vezes em concentrações no estado de equilíbrio para uma dose diária de $30 \mathrm{mg} / \mathrm{dia}(25 \text { a } 670 \mu \mathrm{mol} / \mathrm{L})^{11}$. Esta variabilidade foi explicada pela presença, na população estudada, de metabolizadores lentos e extensivos da esparteína, confirmando que o metabolismo principal da PARO depende da isoenzima CYP2D6. O fato da PARO se ligar a esta isoenzima pode reduzir a clearance de outras drogas administradas concomitantemente, correndo o risco de sua acumulação e de aumento de seus efeitos $\operatorname{adversos}^{12}$.

O objetivo deste estudo foi avaliar o efeito do envelhecimento - incluindo pacientes muito idosos (> 80 anos) - nos níveis plasmáticos de PARO, levandose em conta o gênero e as funções hepática e renal.

\section{Métodos}

\section{Critérios de inclusão e de não-inclusão}

Foram incluídos no estudo os pacientes que viviam na comunidade do Departamento de Psiquiatria Adulta e do Serviço de Psiquiatria Geriátrica da Universidade de Lausanne tratados com PARO e para os quais uma dosagem de rotina clínica desse antidepressivo foi realizada no estado de equilíbrio (pelo menos 2 semanas após a última modificação da dose de PARO e após 12 horas da administração da última dose do medicamento) entre janeiro de 1998 e dezembro de 2002. Os pacientes deviam responder aos critérios diagnósticos da Classificação Estatística Internacional de Doenças (CID-10) de episódio depressivo (F32) ou transtorno depressivo recorrente (F33), conforme estabelecido pelo seu médico assistente.

Não foram incluídos aqueles pacientes que recebiam medicação concomitante capaz de interferir no metabolismo da PARO (em particular, não foram incluídos os pacientes com outros medicamentos metabolizados pelo CYP2D6 do citocromo P450).

Dados foram coletados de 136 pacientes com depressão (44 homens; episódio depressivo CID-10 F32: 48 pacientes; CID-10 F33: 88 pacientes). Os pacientes foram separados em três grupos, com idades diferentes: o primeiro, pacientes com menos do que 65 anos; o segundo, pacientes entre 65 e 79 anos; e o terceiro, pacientes com 80 anos ou mais.

Como as doses de PARO administradas oralmente variaram entre 10 e $120 \mathrm{mg} / \mathrm{dia}$, estas foram normalizadas (matematicamente), assim como os respectivos valores das concentrações plasmáticas (por regra de três), para $20 \mathrm{mg} / \mathrm{dia}$, a fim de poder realizar-se as comparações estatísticas entre os níveis plasmáticos do antidepressivo.

\section{Dosagem bioquímica, estatística}

Os níveis plasmáticos de PARO foram medidos por cromatografia gasosa - espectrometria de massa, como descrito em outros trabalhos ${ }^{13,14}$. As funções hepática e renal foram medidas por testes laboratoriais clínicos estandardizados com um Integra 400 (Roche Diagnostics, Basiléia, Suíça). Comparações das variáveis entre os grupos foram feitas com o teste $t$ de Student e o teste do $\chi^{2}$ quando apropriado. Um valor de $\mathrm{p}$ menor do que 0,05 foi considerado como significativo estatisticamente.

\section{Ética}

O protocolo da pesquisa foi apresentado à Comissão de Ética do Departamento de Psiquiatria Adulta da Universidade de Lausanne em dezembro de 1997, e sua aprovação foi obtida. As dosagens foram realizadas como parte da rotina do curso do tratamento para averiguar as taxas plasmáticas do antidepressivo e correlacioná-las com as respectivas respostas terapêuticas, portanto não foi considerada necessária a obtenção do consentimento escrito dos pacientes para a coleta do sangue - o consentimento oral foi suficiente. 


\section{Resultados}

\section{Dados clínicos}

A Tabela 1 apresenta um resumo dos dados clínicos e dosagens plasmáticas. A proporção de homens e mulheres nos três grupos de pacientes (grupo A: $\leq 64$ anos; grupo B: 65 a 79 anos; grupo C: $\geq 80$ anos) é equivalente $\left(\chi^{2}=1,23\right.$, não-significativa). $\mathrm{A}$ idade média ( \pm desvio padrão) dos pacientes dos três grupos foi de $41,7 \pm 12$ anos, $72,8 \pm 4,7$ anos e $82,8 \pm 3,3$ anos, respectivamente; não foi observada diferença na distribuição do número de pacientes quanto ao sexo entre os três grupos. Não houve diferença dos níveis plasmáticos de creatinina - usada para avaliar a função renal - entre os três grupos de idade. Além disso, não houve também nenhuma diferença significativa nos níveis de transaminase pirúvica (ALT), transaminase oxaloacética (AST) e gama glutamil transpeptidase $(\gamma$ GT), usados para avaliar a função hepática entre os três grupos etários. Os níveis de ALT foram significativamente mais elevados $(\mathrm{p}<0,02)$ nos homens $(33,7 \pm 26,7 \mathrm{IU} / \mathrm{L})$ quando comparados às mulheres $(22,4 \pm 26,2 \mathrm{IU} / \mathrm{L})$, e o valor médio da $\gamma$-GT também foi significativamente maior $(\mathrm{p}<0,05)$ no grupo dos homens $(61,7 \pm 97,8 \mathrm{IU} / \mathrm{L})$ do que no grupo das mulheres $(28,9 \pm 33,2 \mathrm{IU} / \mathrm{L})$. Essas diferenças foram, no entanto, de pequena importância e se encontravam dentro dos limites de normalidade para cada uma dessas substâncias. Pode-se, assim, estimar que, de modo geral, as funções hepática e renal foram equivalentes nos três grupos etários e de pequena importância entre os dois gêneros.

\section{Doses e níveis plasmáticos da PARO}

A média das doses de PARO administradas para todos os pacientes foi de $34,3 \pm 20,1 \mathrm{mg}$. As doses equivalentes administradas para os adultos $(51,4 \pm 23,5$ $\mathrm{mg}$ /dia) foram significativamente superiores $(\mathrm{p}<0,001)$ às doses dadas para os pacientes idosos $(26,3 \pm 10,3 \mathrm{mg} /$ dia) e para os pacientes muito idosos $(22,1 \pm 6,9 \mathrm{mg} /$ dia). A diferença entre a média das doses administradas para os pacientes idosos e muito idosos também foi significativa $(\mathrm{p}<0,05)$.

A Tabela 1 mostra que a média das concentrações plasmáticas da PARO (corrigida para uma dose oral diária de $20 \mathrm{mg}$ ) nos 136 pacientes foi de $43,2 \pm 39,1$ $\mathrm{ng} / \mathrm{mL}$. Para uma mesma dose, houve uma variabilidade interindividual de 37 vezes nos níveis plasmáticos de PARO (concentração máxima/concentração mínima). A média dos níveis plasmáticos de PARO foi significativamente (87\%) mais elevada nos pacientes mais idosos em comparação com os pacientes adultos. A média dos pacientes idosos também foi significativamente mais elevada (57\%) quando comparada com a média dos pacientes adultos. Por outro lado, a média dos níveis plasmáticos de PARO

Tabela 1 - Dados demográficos, laboratoriais, doses diárias de paroxetina administradas e concentrações plasmáticas de paroxetina segundo as diversas categorias etárias, de gênero e total, apresentados como média \pm desvio padrão

\begin{tabular}{|c|c|c|c|c|c|c|c|c|c|c|}
\hline & $\begin{array}{c}\text { Grupo A } \\
<64 \text { anos } \\
(\mathrm{n}=44)\end{array}$ & $\begin{array}{c}\text { Grupo B } \\
65-79 \\
\text { anos } \\
(\mathrm{n}=64)\end{array}$ & $\begin{array}{c}\text { Grupo C } \\
\geq 80 \text { anos } \\
(\mathrm{n}=28)\end{array}$ & $\begin{array}{l}\text { Homens } \\
(\mathrm{n}=44)\end{array}$ & $\begin{array}{l}\text { Mulheres } \\
(\mathrm{n}=92)\end{array}$ & $\begin{array}{l}\text { Todos os } \\
\text { pacientes } \\
(\mathrm{n}=136)\end{array}$ & $\begin{array}{c}\mathrm{A} \\
\text { versus } \\
\mathrm{B}\end{array}$ & $\begin{array}{c}\mathrm{A} \\
\text { versus } \\
\mathrm{C}\end{array}$ & $\begin{array}{c}\mathrm{B} \\
\text { versus } \\
\mathrm{C}\end{array}$ & $\begin{array}{l}\text { Homens } \\
\text { versus } \\
\text { mulheres }\end{array}$ \\
\hline Idade (anos) & $\begin{array}{c}41,7 \\
(12,6)\end{array}$ & $\begin{array}{l}72,8 \\
(4,7)\end{array}$ & $\begin{array}{l}82,8 \\
(3,3)\end{array}$ & $\begin{array}{c}59,4 \\
(19,9)\end{array}$ & $\begin{array}{c}67,9 \\
(16,7)\end{array}$ & $\begin{array}{c}65,4 \\
(18,4)\end{array}$ & $\begin{array}{c}\mathrm{p}< \\
0,001\end{array}$ & $\begin{array}{c}\mathrm{p}< \\
0,001\end{array}$ & $\begin{array}{c}\mathrm{p}< \\
0,001\end{array}$ & $\mathrm{p}<0,02$ \\
\hline Creatinina $(\mu \mathrm{mol} / \mathrm{L})$ & $\begin{array}{c}70,3 \\
(13,1)\end{array}$ & $\begin{array}{c}72,3 \\
(22,2)\end{array}$ & $\begin{array}{c}76,3 \\
(36,9)\end{array}$ & $\begin{array}{c}77,8 \\
(26,8)\end{array}$ & $\begin{array}{c}71,2 \\
(20,1)\end{array}$ & $\begin{array}{c}73,2 \\
(22,1)\end{array}$ & NS & NS & NS & NS \\
\hline $\operatorname{ALT}(\mathrm{IU} / \mathrm{L})$ & $\begin{array}{c}23,2 \\
(15,1)\end{array}$ & $\begin{array}{c}27,9 \\
(39,1)\end{array}$ & $\begin{array}{c}26,3 \\
(12,8)\end{array}$ & $\begin{array}{c}33,7 \\
(26,7)\end{array}$ & $\begin{array}{c}22,4 \\
(26,2)\end{array}$ & $\begin{array}{c}26,3 \\
(26,2)\end{array}$ & NS & NS & NS & $\mathrm{p}<0,02$ \\
\hline AST (IU/L) & $\begin{array}{c}22,3 \\
(10,9)\end{array}$ & $\begin{array}{c}31,3 \\
(48,2)\end{array}$ & $\begin{array}{c}20,4 \\
(13,1)\end{array}$ & $\begin{array}{c}32,3 \\
(47,8)\end{array}$ & $\begin{array}{c}23,1 \\
(20,1)\end{array}$ & $\begin{array}{c}26,1 \\
(29,2)\end{array}$ & NS & NS & NS & NS \\
\hline$\gamma-\mathrm{GT}(\mathrm{IU} / \mathrm{L})$ & $\begin{array}{c}30,8 \\
(41,2)\end{array}$ & $\begin{array}{c}42,1 \\
(56,8)\end{array}$ & $\begin{array}{c}47,9 \\
(68,7)\end{array}$ & $\begin{array}{c}61,7 \\
(97,8)\end{array}$ & $\begin{array}{c}28,9 \\
(33,2)\end{array}$ & $\begin{array}{c}40,1 \\
(54,2)\end{array}$ & NS & NS & NS & $\mathrm{p}<0,05$ \\
\hline Dose média (mg/dia) & $\begin{array}{c}51,4 \\
(23,5)\end{array}$ & $\begin{array}{c}26,3 \\
(10,3)\end{array}$ & $\begin{array}{l}22,1 \\
(6,9)\end{array}$ & $\begin{array}{c}41,8 \\
(26,7)\end{array}$ & $\begin{array}{c}30,3 \\
(14,1)\end{array}$ & $\begin{array}{c}34,3 \\
(20,1)\end{array}$ & $\begin{array}{c}\mathrm{p}< \\
0,001\end{array}$ & $\begin{array}{c}\mathrm{p}< \\
0,001\end{array}$ & $\begin{array}{c}\mathrm{p}< \\
0,05\end{array}$ & $\begin{array}{c}\mathrm{p}< \\
0,001\end{array}$ \\
\hline $\begin{array}{l}\text { Dose mínima-dose } \\
\text { máxima (mg/dia) }\end{array}$ & $20-120$ & $20-60$ & $10-40$ & $20-120$ & $10-80$ & $10-120$ & & & & \\
\hline Paroxetina (ng/mL) & $\begin{array}{c}29,9 \\
(11,9)\end{array}$ & $\begin{array}{c}46,7 \\
(33,4)\end{array}$ & $\begin{array}{c}56,4 \\
(64,1)\end{array}$ & $\begin{array}{c}39,1 \\
(28,3)\end{array}$ & $\begin{array}{c}45,2 \\
(43,2)\end{array}$ & $\begin{array}{c}43,2 \\
(39,1)\end{array}$ & $\begin{array}{c}\mathrm{p}< \\
0,001\end{array}$ & $\begin{array}{c}\mathrm{p}< \\
0,05\end{array}$ & NS & NS \\
\hline
\end{tabular}

NS = não-significativa; ALT $=$ transaminase pirúvica; AST $=$ transaminase oxaloacética; $\gamma$-GT $=$ gama glutamil transpeptidase. 
não diferiu significativamente entre o grupo de muito idosos e de idosos. O resultado de níveis de PARO elevados em pacientes idosos está de acordo com a correlação significativa observada entre a idade e os níveis plasmáticos de PARO $(\mathrm{r}=0,21, \mathrm{p}<0,05)$.

Finalmente, o gênero não influenciou os níveis plasmáticos de PARO, visto que estes valores não foram significativamente diferentes entre os dois sexos, nem no grupo de adultos nem no grupo de idosos. No grupo dos muito idosos, há uma diferença significativa $(t=$ $2,45 ; \mathrm{p}<0,05)$, mas o pequeno número de representantes do sexo masculino nesse grupo $(n=4)$ nos leva a ser prudentes em toda conclusão que se possa tirar desse resultado.

\section{Discussão}

Com o envelhecimento da população, há um risco elevado de crescer o número de idosos com problemas de saúde mental. Entre esses problemas, a depressão é o mais freqüente, com severas conseqüências para a saúde do indivíduo como um todo, implicando inclusive no risco de morte prematura ${ }^{15}$. Apesar de recursos terapêuticos já estarem facilmente disponíveis, a depressão permanece, no grupo de idosos, pouco diagnosticada e, sobretudo, mal tratada. Neste contexto, é então capital evitar uma má escolha do antidepressivo para a obtenção do melhor, mais rápido e mais duradouro resultado terapêutico possível. Entre os fatores determinantes para se escolher um antidepressivo, são citados em ordem de importância decrescente $\left.{ }^{15}: 1\right)$ aspectos farmacocinéticos associados com o envelhecimento; 2) perfil de potenciais efeitos colaterais; 3) interações medicamentosas.

Em conseqüência, estudos farmacocinéticos em pessoas idosas são necessários para se conhecer de forma precisa como esses medicamentos se comportam em organismos envelhecidos. Nos EUA, o relatório de um grupo de peritos ${ }^{16}$ recomendou que os tricíclicos terciários - amitriptilina (AMI), imipramina e doxepina - fossem evitados em idosos. Os ISRS são hoje os antidepressivos mais prescritos para essa faixa da população.

Os idosos se caracterizam por terem uma grande variabilidade interindividual do metabolismo dos medicamentos. Em conseqüência, há uma grande dificuldade para se predizer a dose-alvo para o paciente: isso pode ser demonstrado pela variabilidade interindividual dos níveis plasmáticos encontrada para uma mesma dose padrão do antidepressivo. Num estudo precedente, aplicando a mesma metodologia usada neste atual trabalho, os autores encontraram que a variabilidade interindividual das taxas plasmáticas de AMI em idosos variou até 18 vezes (e a de seu principal metabólito, a nortriptilina, variou 13 vezes) ${ }^{17}$. Em outro estudo semelhante, os autores relataram que, para uma dose de $20 \mathrm{mg}$ de citalopram (CIT), a variabilidade interindividual para suas taxas plasmáticas podia ser de 16 vezes $^{18}$. Esses resultados são bem menores do que a variabilidade de 37 vezes encontrada no presente estudo com a PARO, o que já havia sido confirmado em estudos precedentes ${ }^{10,11}$, mas que não inclúam dados importantes de pacientes com idade muito avançada.

No presente estudo, o gênero não pôde ser responsabilizado por esta variabilidade - a diferença dos níveis de PARO entre os dois sexos foi nãosignificativa, e as funções renal e hepática dos pacientes foram equivalentes nos três grupos do estudo.

A idade foi significativamente correlacionada com os níveis plasmáticos de PARO $(\mathrm{r}=0,21 ; \mathrm{p}<0,05)$. Os níveis plasmáticos de PARO foram $87 \%$ mais elevados no grupo dos pacientes com mais de 80 anos quando comparados com os mesmos níveis nos pacientes adultos, enquanto que eles foram $57 \%$ mais elevados nos pacientes entre 65 e 79 anos em relação aos pacientes adultos. Esses resultados sugerem que os níveis de PARO são aumentados com o envelhecimento, o que foi confirmado por outros autores.

Kaye et al. ${ }^{5}$ constataram que nos idosos existe uma importante variação interindividual nos parâmetros farmacocinéticos no estado de equilíbrio, com aumento estatisticamente significativo das concentrações plasmáticas e diminuição da eliminação.

Lundmark et al. ${ }^{10}$ conduziram um estudo clínico em 21 pacientes com idade média de 72 anos. Encontraram, no estado de equilíbrio, uma considerável variabilidade dos níveis plasmáticos de PARO, com concentrações mais elevadas nos pacientes mais idosos. Igualmente foi observada uma variabilidade significativa da meia-vida de eliminação em idosos. Os autores concluíram que esses resultados não justificavam a necessidade de reduzir as doses da PARO nos idosos e que mais estudos eram necessários.

No estudo com o CIT, encontramos que os níveis plasmáticos de CIT foram 55\% mais elevados no grupo dos pacientes com mais de 80 anos, quando comparados com os mesmos níveis nos pacientes adultos, e 38\% mais elevados nos pacientes entre 65 e 79 anos, em relação aos pacientes adultos. Esses resultados sugerem que os níveis de CIT também são aumentados com o envelhecimento, mas em menor proporção do que a PARO. No entanto, somente um estudo especialmente concebido para comparar os dois antidepressivos responderá se há uma diferença significativa entre eles quanto a esta variabilidade interindividual.

Já no estudo com a AMI, encontramos que os níveis plasmáticos de AMI foram $9 \%$ mais elevados no grupo com mais de 80 anos, quando comparado com os níveis dos pacientes adultos, e equivalentes quando 
comparados com o grupo dos pacientes com idade entre 65 e 79 anos. Concluímos que, globalmente, os níveis plasmáticos de AMI não tinham sido modificados significativamente pelo processo de envelhecimento.

Com relação aos possíveis mecanismos explicativos para o aumento da PARO com o envelhecimento, podemos citar (1) a presença de uma co-medicação prescrita (freqüentemente múltipla em idosos), (2) a diminuição da extração da PARO e/ou (3) o próprio processo de envelhecimento, seja por si só ou associado com a diminuição da extração PARO, via o processo de desmetilação da PARO (mediado pelo CYP2D6) ${ }^{19}$. Quanto à primeira hipótese, a que leva em conta a co-medicação prescrita, os autores não registraram medicação concomitante, o que pode reduzir o valor dos resultados obtidos. No entanto, só foram incluídos na análise pacientes com medicações que sabidamente não interferem com o metabolismo da PARO.

O presente estudo apresenta limitações que podem reduzir o impacto das conclusões. Em primeiro lugar, trata-se de um estudo retrospectivo usando dados medidos para finalidade clínica, e não de pesquisa. Um estudo prospectivo aumentaria em muito a potência das conclusões possíveis. A falta de parâmetros sobre a intensidade do estado depressivo também é limitante. Teria sido útil poder correlacionar as taxas plasmáticas obtidas com o resultado terapêutico. $\mathrm{O}$ mesmo pode ser dito quanto à falta de um registro de efeitos indesejáveis. Esse registro seria valioso para responder, por exemplo, à questão de se saber se simultaneamente ao aumento dos níveis plasmáticos da PARO havia um aumento de efeitos indesejáveis. Finalmente, um registro fiel da co-medicação teria facilitado o estudo dos potenciais riscos de interação medicamentosa, em particular no contexto de aumento dos níveis plasmáticos.

\section{Conclusão}

O presente estudo confirma a observação da existência de uma significativa variabilidade interindividual dos níveis plasmáticos de PARO para uma dose determinada. A idade contribui em parte para esta variabilidade, enquanto que o gênero não a influencia. Um aumento médio de 57 a $87 \%$ dos níveis plasmáticos de PARO foram medidos em pacientes idosos e muito idosos em comparação com estes níveis em pacientes mais jovens. Uma redução da dose de PARO em pacientes idosos (com mais de 65 anos) é recomendada, mas não há consenso quanto a isso. Apesar de não haver nenhuma evidência da existência de uma relação entre um intervalo de concentração plasmática ideal e resposta terapêutica máxima ou entre concentração plasmática crítica e risco aumentado de efeitos adversos, o monitoramento dos níveis plasmáticos parece justificado quando consideramos as observações feitas neste estudo e ao se levar em conta a possível sensibilidade aumentada dos pacientes idosos aos medicamentos, também por fatores farmacodinâmicos. Os limites deste estudo nos levam a propor novas pesquisas com metodologia mais apurada, mas incluindo também um número expressivo de pacientes com mais de 80 anos.

\section{Agradecimentos}

Os autores agradecem ao Dr. Michel Gaillard pelo apoio na realização e execução deste estudo.

\section{Referências}

1. Alexopoulos GS, Katz IR, Reynolds CF 3rd, Carpenter D, Docherty JP. Pharmacotherapy of depressive disorders in older patients. Postgrad Med. 2001;Spec $\mathrm{N}^{\circ}$ Pharmacotherapy:1-86.

2. Reynolds CF 3rd, Dew MA, Pollock BG, Mulsant BH, Frank E, Miller MD, et al. Maintenance treatment of major depression in old age. N Engl J Med. 2006;354(11):1130-8.

3. Hiemke C. [Paroxetine: pharmacokinetics and pharmacodynamics] Fortschr Neurol Psychiatr. 1994;62(Suppl 1):2-8.

4. Bayer AJ, Roberts NA, Allen EA, Horan M, Routledge PA, Swift $\mathrm{CG}$, et al. The pharmacokinetics of paroxetine in the elderly. Acta Psychiatr Scand Suppl. 1989;350:85-6.

5. Kaye CM, Haddock RE, Langley PF, Mellows G, Tasker TC, Zussman BD, et al. A review of the metabolism and pharmacokinetics of paroxetine in man. Acta Psychiatr Scand Suppl. 1989;350:60-75.

6. Dechant KL, Clissold SP. Paroxetine: a review of its pharmacodynamic and pharmacokinetic properties, and therapeutic potential in depressive illness. Drugs. 1991;41(2):225-53.

7. Tulloch IF, Johnson AM. The pharmacologic profile of paroxetine, a new selective serotonin reuptake inhibitor. J Clin Psychiatry. 1992;53(Suppl):7-12.

8. Bloomer JC, Woods FR, Haddock RE, Lennard MS, Tucker GT. The role of cytochrome P4502D6 in the metabolism of paroxetine by human liver microsomes. Br J Clin Pharmacol. 1992;33(5):5213 .

9. Dunner DL. An overview of paroxetine in the elderly. Gerontology. 1994;40(Suppl 1):21-7.

10. Lundmark J, Scheel Thomsen I, Fjord-Larsen T, Manniche PM, Mengel H, Moller-Nielsen EM, et al. Paroxetine: pharmacokinetic and antidepressant effect in the elderly. Acta Psychiatr Scand Suppl. 1989;350:76-80.

11. Sindrup SH, Brosen K, Gram LF. Pharmacokinetics of the selective serotonin reuptake inhibitor paroxetine: nonlinearity and relation to the sparteine oxidation polymorphism. Clin Pharmacol Ther. 1992;51(3):288-95.

12. Catterson ML, Preskorn SH. Pharmacokinetics of selective serotonin reuptake inhibitors: clinical relevance. Pharmacol Toxicol. 1996;78(4):203-8.

13. Eap CB, Baumann P. Analytical methods for the quantitative determination of selective serotonin reuptake inhibitors for therapeutic drug monitoring purposes in patients. J Chromatogr B Biomed Appl. 1996;686(1):51-63.

14. Eap CB, Bouchoux G, Amey M, Cochard N, Savary L, Baumann P. Simultaneous determination of human plasma levels of citalopram, paroxetine, sertraline, and their metabolites by gas chromatographymass spectrometry. J Chromatogr Sci. 1998;36(7):365-71.

15. Baldwin RC, Chiu E, Katona C, Graham N. Guidelines on 
depression in older people. Practising the evidence. London: Martin Dunitz; 2002.

16. Mulsant BH, Alexopoulos GS, Reynolds CF III, Katz IR, Abrams R, Oslin D, et al. Pharmacological treatment of depression in older primary care patients: the PROSPECT algorithm. Int J Geriatr Psychiatry. 2001;16(6):585-92.

17. Lima CAM. Efeitos da idade, do sexo e do índice de massa corporal nas concentrações plasmáticas de amitriptilina e nortriptilina em pacientes adultos e idosos. Arq Bras Psiquiatr Neurol Med Legal.
2006;100(2):17-23.

18. de Mendonça Lima CA, Baumann P, Brawand-Amey M, Brogli C, Jacquet S, Cochard N, et al. Effect of age and gender on citalopram and desmethylcitalopram steady-state plasma concentrations in adults and elderly depressed patients. Prog Neuropsychopharmacol Biol Psychiatry. 2005;29(6):952-6.

19. Feng Y, Pollock BG, Ferrell RE, Kimak MA, Reynolds CF 3rd, Bies RR. Paroxetine: population pharmacokinetic analysis in late-life depression using sparse concentration sampling. $\mathrm{Br} \mathrm{J}$ Clin Pharmacol. 2006;61(5):558-69. 